\title{
Pretreatment Diagnosis of Suprasellar Papillary Craniopharyngioma and Germ Cell Tumors of Adult Patients
}

\author{
(D) H.-J. Lee, C.-C. Wu, H.-M. Wu, S.-C. Hung, J.-F. Lirng, C.-B. Luo, F.-C. Chang, and W.-Y. Guo
}

\begin{abstract}
BACKGROUND AND PURPOSE: Suprasellar papillary craniopharyngiomas and germ cell tumors in adults share some clinical and imaging similarities but have different therapeutic strategies and outcomes. This study aimed to evaluate the pretreatment diagnosis of these 2 tumors to improve the therapeutic outcome.
\end{abstract}

MATERIALS AND METHODS: We retrospectively enrolled 18 adults with papillary craniopharyngiomas and 17 with germ cell tumors. The MR imaging findings were evaluated, including signal change and anatomic extension. The medical records were reviewed to collect clinical findings, management, and outcomes.

RESULTS: The clinical findings of papillary craniopharyngiomas versus germ cell tumors were as follows: age: $46 \pm 13.9$ years versus $23 \pm$ 7.1 years $(P<.0001)$; diabetes insipidus: $2 / 18(11 \%)$ versus $11 / 17(65 \%)(P=.001)$; recurrence $13 / 16(81 \%)$ versus $4 / 17(24 \%)(P=.0031)$. The MR imaging findings of papillary craniopharyngiomas versus germ cell tumors were as follows_pituitary stalk thickening: $1.6 \pm 0.4 \mathrm{~mm}$ versus $5.4 \pm 4.2 \mathrm{~mm}(P<.0001)$; vertical infundibular extension: 1/18 (6\%) versus $16 / 17(94 \%)(P<.0001)$; sagittal spheric shape: $17 / 18(94 \%)$ versus $1 / 17(6 \%)(P<.0001)$; diffusion restriction: $1 / 17(6 \%)$ versus 8/12 (67\%) $(P=.0009)$.

CONCLUSIONS: Younger age, diabetes insipidus, MR imaging characteristics of restricted diffusion, and vertical infundibular extension favor the diagnosis of germ cell tumors. Spheric shape without infundibular infiltration provides clues to papillary craniopharyngiomas, which originate from the pars tuberalis and are located outside the third ventricle. We suggest that suprasellar germ cell tumor is possibly an intraventricular lesion. Appropriate treatment planning can be initiated according to the diagnosis and anatomic location.

ABBREVIATIONS: $\mathrm{GCT}=$ germ cell tumor; $\mathrm{PCP}=$ papillary craniopharyngioma

E xcept for pituitary macroadenoma, suprasellar tumors are infrequent in adult patients. Some of these suprasellar tumors have specific clinical and imaging features that make them straightforward to diagnose and subsequently manage. These classic features include a dural tail appearance in meningioma; young age; cysts and calcifications in adamantinomatous cranio-

Received July 3, 2014; accepted after revision August 17.

From the Department of Radiology, Taipei Veterans General Hospital, Taipei, Taiwan; and School of Medicine, National Yang-Ming University, Taipei, Taiwan.

This work was supported in part by grants from Taipei Veterans General Hospital (V103C-063, Cl-103-3) and the Ministry of Science and Technology (NSC 102-2314-B-075-068).

Paper previously presented in part at: Annual Meeting of the Japanese Society of Neuroradiology, March 21-23, 2014; Yonago, Japan.

Please address correspondence to Feng-Chi Chang, MD, Department of Radiology, Taipei Veterans General Hospital, 201 Shih-Pai Rd, Sec 2, Taipei, Taiwan; e-mail: fcchang374@gmail.com

- Indicates open access to non-subscribers at www.ajnr.org

三 Indicates article with supplemental on-line table.

http://dx.doi.org/10.3174/ajnr.A4142 pharyngioma; homogeneous infiltrating mass of hypothalamic/optic pathway gliomas; a midline enhancing lesion in germ cell tumor (GCT); specific locations with signal similar to those of gray matter in hamartoma; and bright signals on DWI in epidermoids. ${ }^{1-3}$ However, some suprasellar tumors in adults can mimic each other in some clinical and imaging features, making pretreatment planning difficult.

Papillary craniopharyngiomas (PCPs) and GCTs are 2 unusual suprasellar tumors in adults, with similar clinical and imaging findings in some aspects. Clinically, PCPs and GCTs mostly occur in young adults with visual impairment or pituitary axis dysfunction or diabetes insipidus. They commonly present as predominantly solid or mixed cysts and solid suprasellar lesions with contrast enhancement on MR imaging. However, the treatment and outcomes of both tumor types are quite different. ${ }^{2,4,5}$ Surgical resection not only is the main treatment of PCPs but also remarkably affects the outcome. ${ }^{6}$ By contrast, complete surgical resection is usually unnecessary in patients with germinoma. This radiosensitive tumor can be well-controlled by radiation therapy alone or 
Table 1: Analysis of clinical symptoms and outcomes of suprasellar papillary craniopharyngioma and suprasellar germ cell tumors

\begin{tabular}{|c|c|c|c|c|c|}
\hline Variables & $\operatorname{PCP}(n=18)$ & $\mathrm{GCT}(n=17)$ & OR & $95 \% \mathrm{Cl}$ & $P$ Value ${ }^{\text {a }}$ \\
\hline Age (yr) & $46 \pm 13.9(21-70)$ & $23 \pm 7.1(16-43)$ & & & $<.0001$ \\
\hline Sex (male:female) & 13:5 & $13: 4$ & 1.3 & $.27-5.7$ & .7738 \\
\hline \multicolumn{6}{|l|}{ Symptoms } \\
\hline Visual field deficits & $12(67 \%)$ & $6(35 \%)$ & .27 & $.07-1.1$ & .0634 \\
\hline Hypopituitarismb & $12(71 \%)$ & $12(75 \%)$ & 1.3 & $.27-5.8$ & .7761 \\
\hline Hyperprolactinemia $^{c}$ & $14(93 \%)$ & $11(67 \%)$ & .16 & $.02-1.5$ & .0834 \\
\hline Hypothalamic dysfunction, including diabetes insipidus & $2(11 \%)$ & $11(65 \%)$ & 15 & $2.5-87$ & .0010 \\
\hline Initial management & & & & & .0303 \\
\hline Total resection & $7(39 \%)$ & $3(18 \%)$ & 1.0 & - & \\
\hline Subtotal resection & $6(33 \%)$ & $1(6 \%)$ & .39 & $.03-4.8$ & \\
\hline Partial resection or biopsy & $3(17 \%)$ & $6(35 \%)$ & 4.7 & $.67-32$ & \\
\hline Radiation or radiosurgery & $2(11 \%)$ & $7(41 \%)$ & 8.2 & $1.03-65$ & \\
\hline \multicolumn{6}{|l|}{ Outcome } \\
\hline Recurrence $^{d}$ & $13(81 \%)$ & $4(24 \%)$ & .10 & $.02-.50$ & .0031 \\
\hline Survival (mean) & $39.6 \pm 32.02(2-118)$ & $52.4 \pm 40.32(13-123)$ & & & .3815 \\
\hline
\end{tabular}

a $P=$ statistical significance as calculated by the log-rank test.

${ }^{\mathrm{b}}$ In the PCP group, $n=17$; in the GCT group, $n=16$.

' In the PCP group, $n=15$; in the GCT group, $n=16$.

${ }^{\mathrm{d}}$ In the PCP group, $n=16$; in the GCT group, $n=17$

combined with chemotherapy, even without a tissue diagnosis. ${ }^{7}$ The preoperative diagnosis of these 2 unusual suprasellar tumors in adults facilitates appropriate treatment planning and avoids associated surgical morbidity. We presumed that the distinct embryology and pathogenesis of the 2 tumors develop unique clinical and MR imaging characteristics. This study was designed to evaluate retrospectively the clinical and MR imaging findings of suprasellar papillary craniopharyngioma and germ cell tumors in adult patients, to obtain a pretreatment diagnosis.

\section{MATERIALS AND METHODS Study Population}

This retrospective study was reviewed by an institutional review board. Between 2003 and 2013, 58 patients with craniopharyngioma and 17 patients with germ cell tumors who were older than 16 years of age were diagnosed at our institution. Of the 58 pathologically diagnosed craniopharyngiomas, 21 adamantinomatous craniopharyngiomas and 12 in patients without mention of specific subtypes or mixed type of adamantinomatous and papillary components were excluded. Seven patients with PCPs with incomplete preoperative MR imaging studies were also excluded. We excluded the patients with adamantinomatous craniopharyngiomas because they can usually be diagnosed by their classic imaging findings. ${ }^{8}$ Thus, 18 patients with PCP were included in the present study. Among the 17 consecutive patients with a GCT at a suprasellar location, 8 were pathologically diagnosed with a germinoma; 1 , with an immature teratoma; 1 , with a mixed germ cell tumor; and 7 patients with germinoma were diagnosed on the basis of clinical and neuroradiologic features (Table 1). ${ }^{9}$ The medical records of both groups were reviewed to collect demographic information, presenting symptoms, laboratory data, management information, and outcome information.

\section{Clinical Findings}

We included the symptoms of visual field defects, hypopituitarism, and hypothalamic dysfunction. Visual field tests were analyzed by ophthalmologic examination. Hypopituitarism included symptoms of orthostatic hypotension, amenorrhea, impotence, or a decreased level of hypothalamic-pituitary axis hormones
(Table 1). Hypothalamic dysfunction included symptoms of diabetes insipidus or weight gain. The level of prolactin, $\beta$-human chorionic gonadotropin, and $\alpha$-fetoprotein were also assessed.

\section{MR Imaging Findings}

MR images were retrospectively blindly reviewed by 2 certified neuroradiologists (F.-C.C. and H.-J.L). All quantitative measurements were made by standard tools in the hospital PACS, and qualitative assessments of lesional characters were made on the basis of consensus. We evaluated the MR imaging findings of the 2 suprasellar tumors regarding the location, extent (largest sagittal diameter), components, signal, shape, involvement of the pituitary stalk and pituitary gland, and other associated findings (Online Table). The locations of the tumors were divided into 3 subgroups: those located at the suprasellar space and extending toward the third ventricle; those located at the suprasellar region with sellar extension, causing displacement of the pituitary gland; and those involving the sellar and suprasellar spaces and third ventricle. The size of the tumor was measured as the largest diameter at the midsagittal plane on contrast-enhanced T1WI. If the solid component of the tumor exceeded $50 \%$ of the total tumor volume on both sagittal and coronal imaging, the tumor was defined as having a solid predominance and vice versa. The signal intensities of the solid part of the tumors were further compared with those of the gray matter at the adjacent temporal lobe on T1WI, T2WI, and DWI. The signal changes on noncontrast and post-contrast-enhanced T1WI were also evaluated. The patterns of contrast enhancement were further described as enhancement if the solid component showed strong enhancement and marginal enhancement if there was peripheral enhancement along the margin.

The shape of the tumors was evaluated on sagittal imaging. If the lower end of the tumor appeared as a round or blunted shape in the suprasellar region, the tumor was described as spheric (Figs $1 A$ and $2 A$ ). If the low end of the tumor extended vertically down to the sellar region, the tumor was described as transinfundibular (Figs $3 A$ and $4 B) .{ }^{10}$ The diameter of the pituitary stalk was measured at the narrowest part on contrast-enhanced sagittal T1WI. 

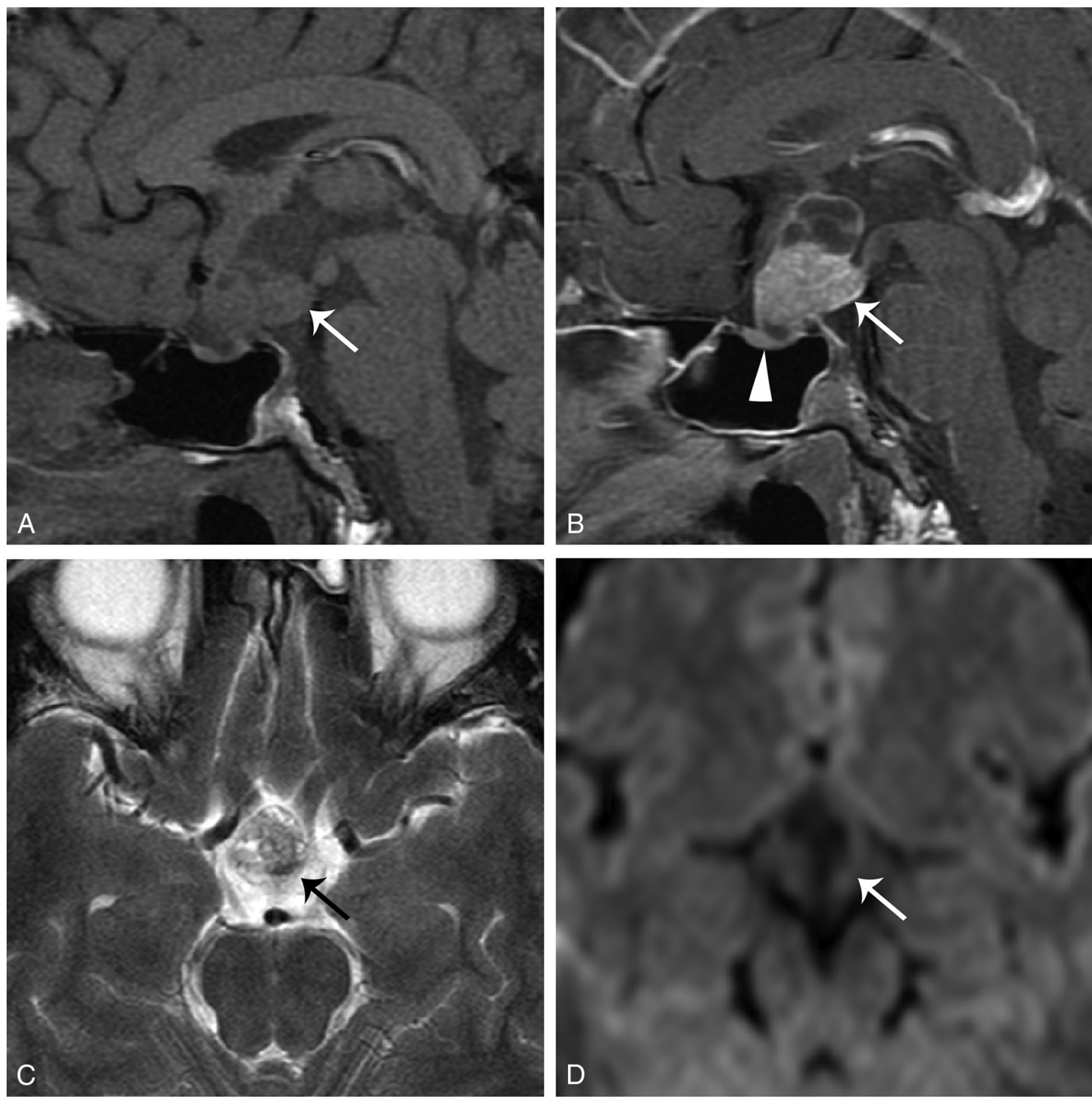

FIG 1. A 28-year-old man had bitemporal hemianopsia for several weeks. Surgical resection revealed PCP. A, Sagittal TIWI shows a mixed solid and cystic tumor (arrow) at the sellar and suprasellar regions. B, Contrast-enhanced sagittal TTWI shows strong enhancement at the solid component and its cystic wall (arrow). The lower end of the tumor is spheric. The pituitary gland is compressed but intact (arrowhead). C, Axial T2WI demonstrates that the tumor (arrow) has mixed isointense-to-hyperintense signals compared with the adjacent temporal gray matter. $D$, DWI $(b=800)$ shows no restricted diffusion in the tumor (arrow).

If the diameter of the infundibulum was $\geq 4 \mathrm{~mm}$, infundibular thickening was defined. ${ }^{11}$ The relationship between the tumor and the pituitary gland was described as infiltrative if there was an indistinct and irregular border between the tumor and the gland on sagittal and coronal imaging (Figs 3 and $4 A,-B$ ). When the pituitary gland was compressed but intact, it was described as a compression (Fig 1B). The associated findings of involvement of the optic pathway and hypothalamus and dilation of the ventricles were analyzed (On-line Table).

Except for the 2 patients with GCT who underwent surgical resection, all patients of both groups underwent radiation therapy of the tumor bed or residual tumor and/or the ventricular system.
To check the relationship between suprasellar GCT and the possible origin of the pineal region, we evaluated the interval changes of the pineal gland and suprasellar tumor of both groups. The largest diameter of the pineal gland and/or its associated tumor on contrast-enhanced sagittal T1WI in the initial studies was compared with those in the latest follow-up series, to evaluate the effect of radiation therapy on the pineal gland or its associated tumor (mean, 36.8 \pm 31.2 months). If the largest diameter changed $>25 \%$ from the initial one, a significant interval change of volume was defined. The presence of ventricular or subarachnoid tumor seeding was evaluated by contrast-enhanced T1WI. 

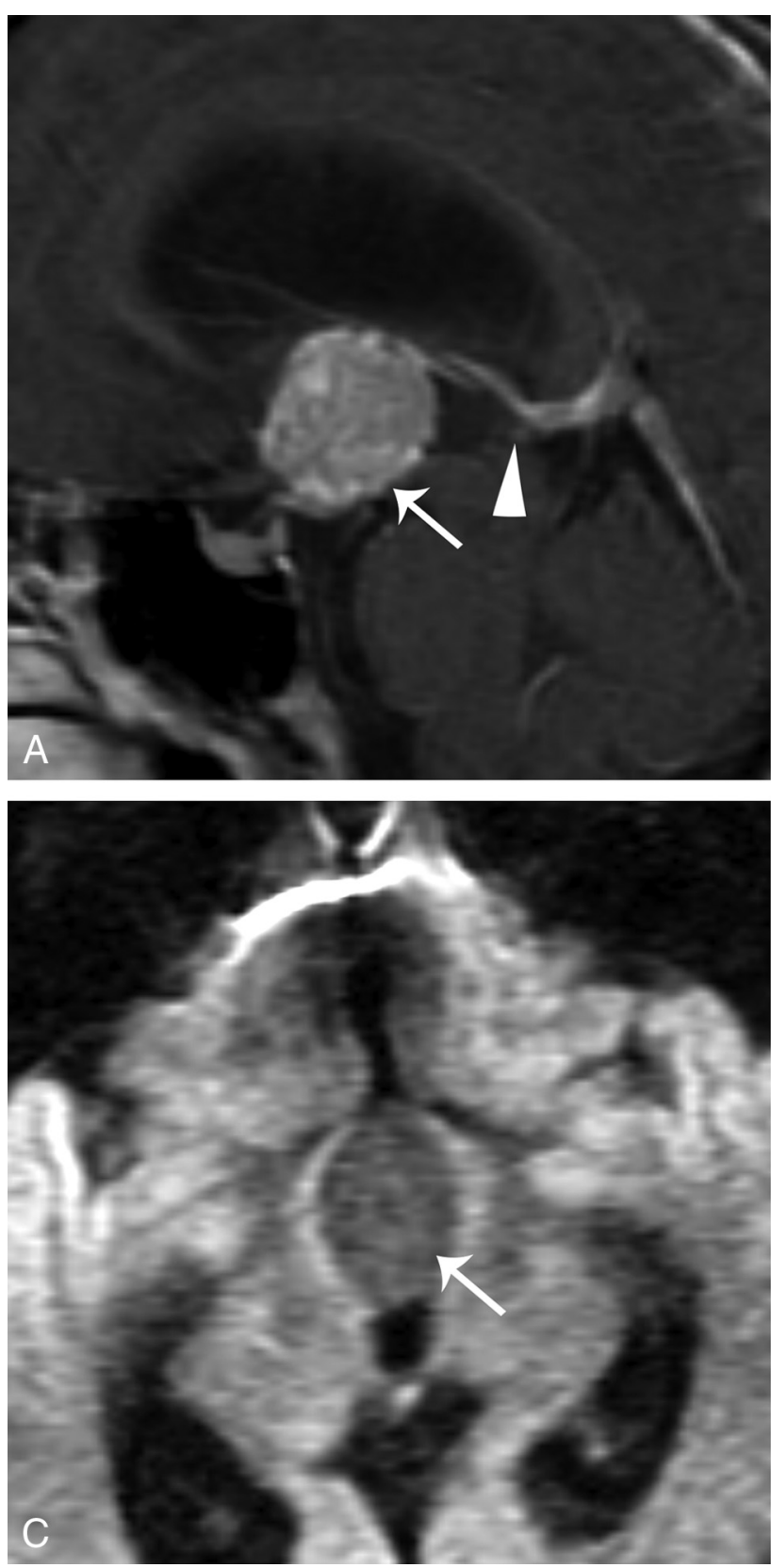

\section{Management and Outcome}

Initial management was determined according to the preoperative diagnosis by the clinical and neuroradiologic findings. The surgical approach included craniotomy or transsphenoidal resection aimed at obtaining tumor removal in all patients with PCP and in 7 with GCT. Postoperative radiation therapy of the residual tumor/tumor bed and/or ventricular system was assigned for both tumor types. Radiation therapy was the initial treatment in 10 patients with GCT when they had a tissue or a clinical diagnosis of germinoma. The initial results of surgical resection were classified as gross total removal, subtotal removal, and partial removal or biopsy of the tumor, which were defined as no tumor left under maximum magnification, residual invaded parenchyma, and persistent macroscopic fragments, respectively. ${ }^{12}$ Complications related to the management and any tumor recurrence were recorded to evaluate the outcome. Radiation therapy or gamma

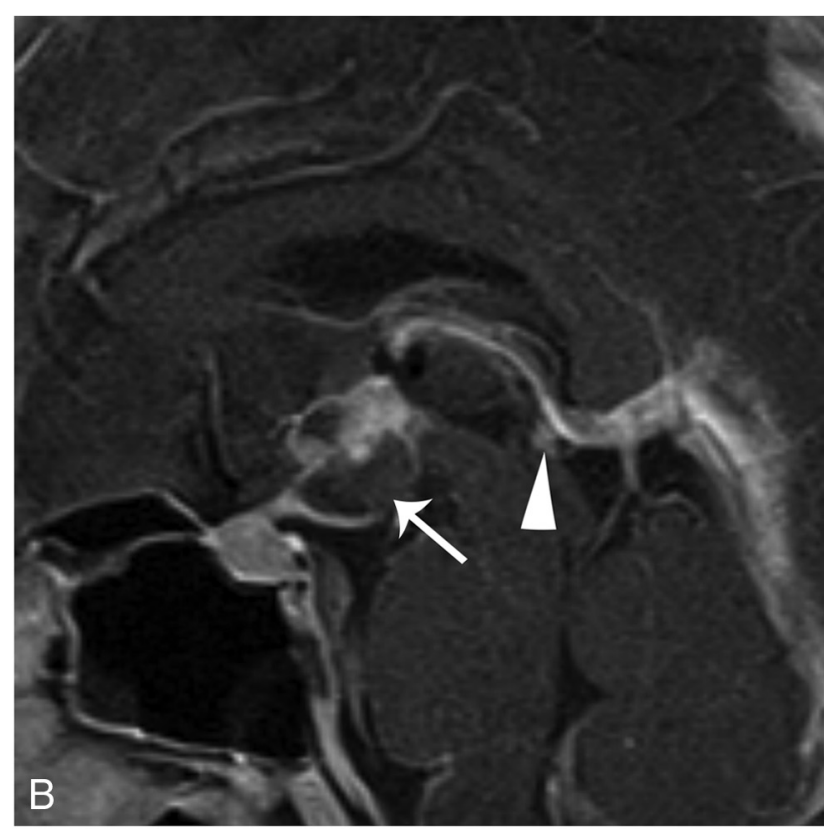

FIG 2. A 38-year-old man had intermittent dizziness for 2 months and right-sided limb weakness for 1 week. Surgical resection revealed PCP. Brain MR imaging. A, Contrast-enhanced sagittal TTWI shows a heterogeneous enhancing tumor (arrow) at the suprasellar region and third ventricular floor. The lower end of the tumor is spheric. The size of the pineal gland is within normal range (arrowhead). B. The 2.5-year follow-up contrast-enhanced sagittal TIWI shows a mixed cystic and solid recurrent tumor (arrow). The size of the pineal gland (arrowhead) remains unchanged. C, DWI $(b=800)$ reveals no restricted diffusion in the tumor (arrow). knife radiosurgery was conducted for recurrent tumor. The survival time was also calculated.

\section{Statistical Analysis}

We analyzed the clinical and MR imaging findings of both groups. Demographic features, clinical presentations, management and outcome, and MR imaging findings were examined by using the Fisher exact test. The odds ratios and the accompanying 95\% confidence intervals were calculated. For all analyses, $P<.05$ was statistically significant.

\section{RESULTS}

\section{Clinical Findings}

The demographic and clinical findings of both groups are summarized in Tables 1 and 2. The age of patients with GCT was significantly younger than that in those with PCP $(P<.0001)$. 

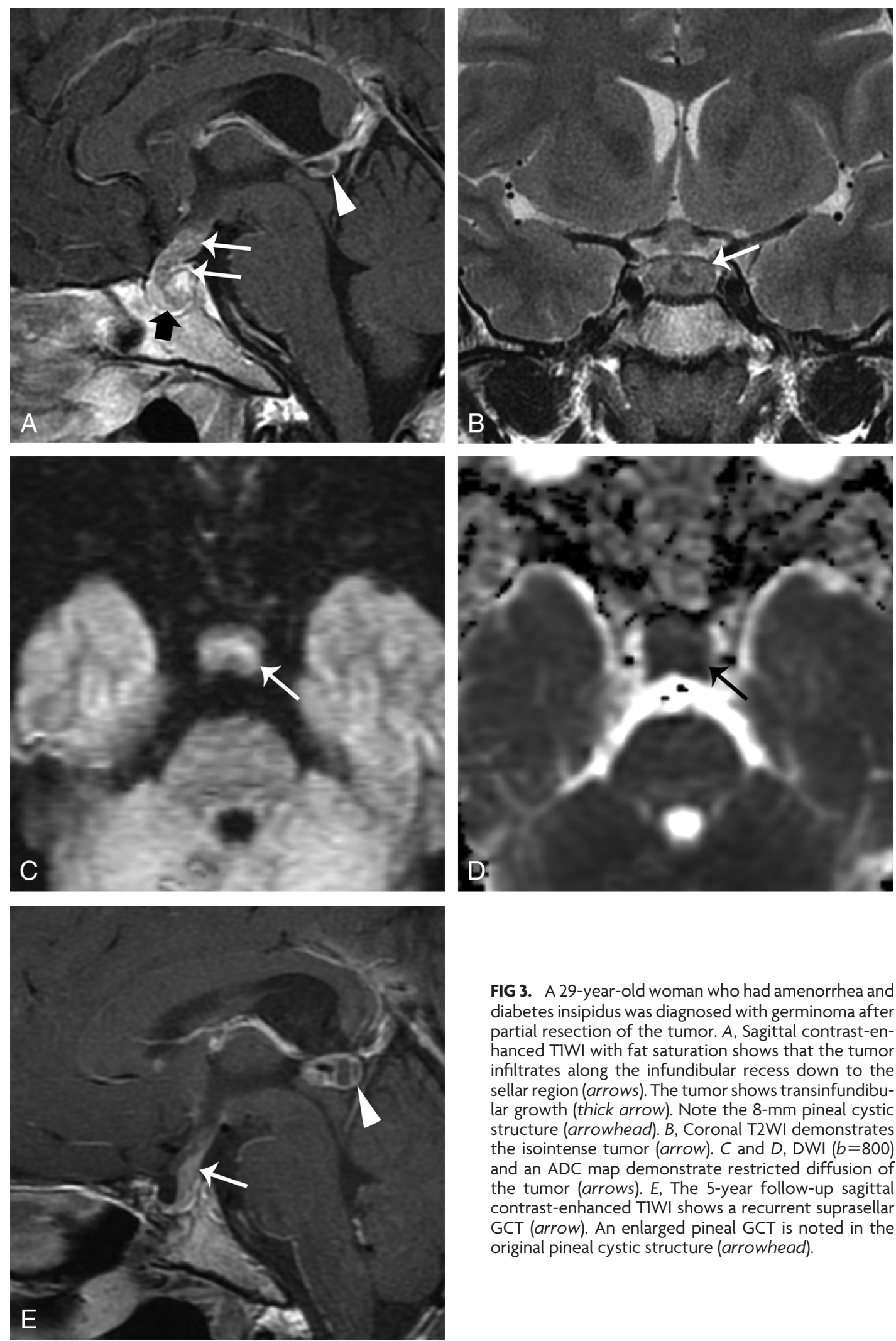

FIG 3. A 29-year-old woman who had amenorrhea and diabetes insipidus was diagnosed with germinoma after partial resection of the tumor. A, Sagittal contrast-enhanced TIWI with fat saturation shows that the tumor infiltrates along the infundibular recess down to the sellar region (arrows). The tumor shows transinfundibular growth (thick arrow). Note the 8-mm pineal cystic structure (arrowhead). B, Coronal T2WI demonstrates the isointense tumor (arrow). $C$ and D, DWI $(b=800)$ and an ADC map demonstrate restricted diffusion of the tumor (arrows). E, The 5-year follow-up sagittal contrast-enhanced TIWI shows a recurrent suprasellar GCT (arrow). An enlarged pineal GCT is noted in the original pineal cystic structure (arrowhead).

The symptoms related to an impaired visual field and pituitary dysfunction were commonly found in both groups but showed no significant difference. Hypothalamic dysfunction and diabetes insipidus were significantly associated with patients with GCT $(P=.001$; OR, 15; 95\% CI, 2.5-87). Besides the $7 / 17$
(41\%) patients in the GCT group diagnosed clinically, $\alpha$-fetoprotein and $\beta$-human chorionic gonadotropin levels were examined in $2 / 10(20 \%)$ patients of the GCT group and $4 / 18$ (22\%) patients of the PCP group before the operation. $\alpha$-fetoprotein levels were elevated in 2 patients, including 1 with 

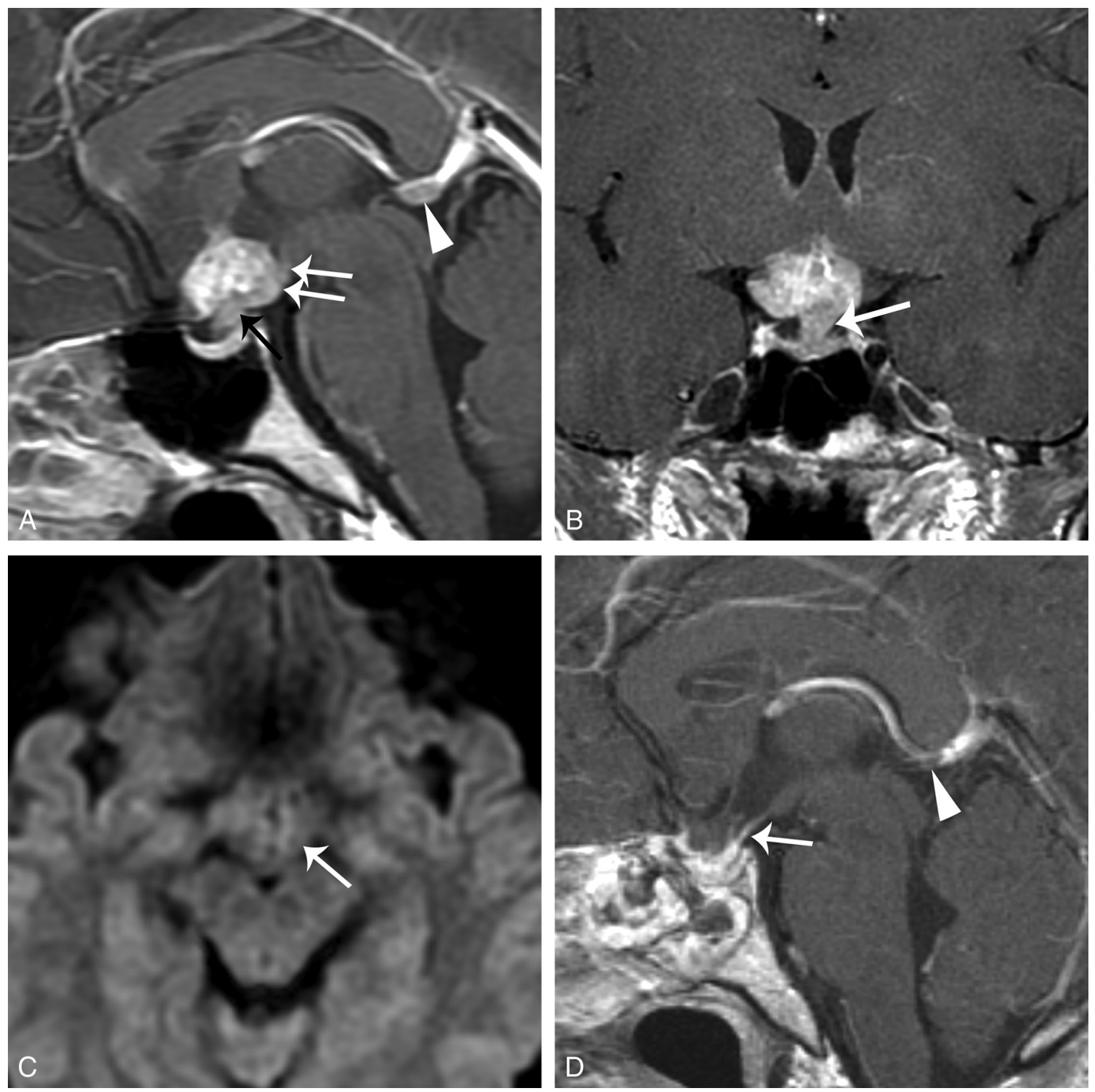

FIG 4. A 23-year-old woman had amenorrhea for 2 years and biopsy-proved germinoma. A, Sagittal contrast-enhanced TIWI with fat saturation shows a heterogeneous enhancing suprasellar and third ventricular floor tumor (white arrows) with infundibular stalk thickening (black arrow). The pineal gland is $8 \mathrm{~mm}$ in the largest diameter (arrowhead). B. Coronal contrast-enhanced TTWI shows the growth of the tumor along the infundibular recess (arrow) and the indistinct margin between the tumor and pituitary gland. C, DWI $(b=800)$ demonstrates mildly high signal in the tumor (arrow). D, The 3-month follow-up sagittal contrast-enhanced T7WI after surgical and radiation treatment shows shrinkage of both the suprasellar (arrow) and pineal tumors (arrowhead).

mixed germ cell tumor and 1 with immature teratoma; $\beta$-human chorionic gonadotropin levels were elevated in 1 patient with immature teratoma and 2 with germinomas.

\section{MR Imaging Findings}

MR imaging findings of both groups are summarized in the Online Table and Table 2. Both suprasellar tumors could extend to the sellar region and third ventricle. PCPs had a larger size than GCTs at the time of diagnosis, with a mean diameter of $3.18 \mathrm{~cm}$ on sagittal images $(P=.0064)$. Compared with the predominantly heterogeneous solid characteristics in the GCT groups, PCPs were mostly well-circumscribed mass lesions with heterogeneous, multicystic components ( $P=.0002$; OR, 0.03 ; 95\% CI, $0.003-$ 0.30). The multicystic components of PCPs showed prominent marginal enhancement on contrast-enhanced T1WI compared with the heterogeneous strong enhancement of the solid portion of GCTs $(P<.0001)$. Most of the solid parts of both tumors showed isointense signal intensity in the gray matter on T1WI and T2WI, which demonstrated no significant difference $(P=.2962$ and 0.9616 , respectively). On DWI, the solid parts of GCTs revealed higher signal intensity than the adjacent gray matter, and PCPs had lower signal intensity than the gray matter. The signal 


\begin{tabular}{|c|c|c|}
\hline \multicolumn{3}{|l|}{ Clinical findings } \\
\hline Hypothalamic dysfunction, including diabetes insipidus & - & Yes \\
\hline \multicolumn{3}{|l|}{ MR imaging characteristics } \\
\hline Component & Cystic predominance & Solid predominance \\
\hline DWI signals in the solid part & Hypointense & Isointense \\
\hline Marginal contrast enhancement & Yes & - \\
\hline Shape & Spheric & Transinfundibular \\
\hline Pituitary stalk & - & Thickening \\
\hline Tumor seedings & - & Yes \\
\hline Size change of the pineal gland after radiotherapy & - & Yes \\
\hline Main management strategy & Surgery & Radiation therapy \\
\hline Outcomes & Higher recurrence rate & - \\
\hline
\end{tabular}

Note:-indicates the feature was significantly lower or less in the specific tumor group.

intensity was significantly different between both tumor groups $(P=.0009)$.

Regarding the shape of the lower part of both tumors on sagittal images, PCPs appeared spheric or blunted in all except 1 patient; additionally, the GCT extended vertically downward along the pituitary stalk in all except 1 patient $(P<.0001$; OR, 272; 95\% CI, 16-4724). Infundibular thickening was observed in 13 of 17 GCTs, and the mean diameter of the pituitary stalk was $5.4 \mathrm{~mm}$. Infundibular thickening was not noted in patients with PCPs with a mean value of $1.6 \mathrm{~mm}(P<.0001)$. Although patients with GCTs involving the pituitary gland were more frequent than those with PCPs, no significant difference was found between the 2 groups $(P=.1896)$.

Seeding lesions in the subarachnoid space or ventricle were noted in 8 patients with GCT and in none with PCP $(P=.0009)$. Except for 6 patients with PCP, all the other patients of both groups underwent radiation therapy. Thereafter, a significant interval change of the size of the pineal gland was noted in 8 of the 16 patients with GCT (50\%), but in none in the patients with PCP $(P=.0009)$. These included 7 patients with a reduced size (Fig $4 D$ ) and 1 patient with an increased size of the pineal gland (Fig $3 E$ ). In both tumor types, some of the optic tract and hypothalamus had edema. However, the PCP group demonstrated more significant compression and displacement of the optic chiasm compared with the GCT group ( $P=.0019$; OR, 0.05; 95\% CI, 0.01-0.49).

\section{Management and Outcome}

For patients with PCP, the extent of the initial resection was grossly total in 7 patients, subtotal in 6 patients, and partial or only a biopsy in 3 patients. The other 2 patients with PCP underwent radiation therapy initially because the preoperative impression was GCT. They had surgical resection due to poor response to radiation therapy and local recurrence. One underwent a subtotal resection 10 months after initial radiation therapy; the other underwent 3 operations and 1 session of radiosurgery in 10 years. Regarding the treatment outcomes, 2 surgery-related deaths occurred in the PCP group $(2 / 18,11 \%)$. One was caused by intracranial infection 6 days after the surgery; the other patient died of severe extrapontine myelinolysis 1 month after the surgery. Of the other 16 patients in the PCP group, 13 (81\%) had residual or recurrent tumors that required adjuvant radiation therapy, radiosurgery, or repeat surgery. Only 3 of the 16 patients (19\%) in the PCP group obtained complete tumor removal after initial management.
Ten of the 17 patients of the GCT group received surgical treatment. The surgical results were total tumor removal in $3 \mathrm{pa}-$ tients, subtotal resection in 1 patient, and partial resection or biopsy in 6 patients. Four of the 6 patients had a partial resection or biopsy because the diagnosis of GCT was made on frozen section pathology. The other 7 patients with GCT diagnosed by clinical and MR imaging findings underwent radiation therapy as the initial treatment. No mortality in the GCT group was noted.

After initial management, the recurrence rate in the PCP and GCT groups was $81 \%$ versus $24 \%$, respectively $(P=.003$; OR, 0.10 ; $95 \%$ CI, 0.02-0.50). Repeat surgery or adjuvant radiosurgery was performed for the 12 patients with PCP with recurrent tumors. In the GCT group, only 1 patient had recurrent tumors after surgery and radiation therapy. Although the pineal gland was initially normal, recurrent tumors were observed in both the infundibular and pineal regions (Fig 2E). The survival interval was longer in patients with GCT (52.4 \pm 40.3 months) than in those with PCP $(39.6 \pm 32.0$ months $)(P=.3815)$, but it was not statistically significant.

\section{DISCUSSION}

The pituitary gland is divided into 2 anatomic compartments from different ectodermal origins. At the fourth week of gestation, the Rathke pouch forms from the evagination of an ectodermal outpouching of stomodeum immediately in front of the buccopharyngeal membrane. The cells in the anterior wall of the Rathke pouch proliferate rapidly to form the pars anterior. The less active cells in the posterior wall form the pars intermedia. By the 16th week of gestation, the pars tuberalis, a small part of the pars anterior, extends superoventrally and wraps around the pituitary stalk (Fig 5A). ${ }^{13,14}$ It was hypothesized that PCPs originated from squamous epithelial cells in the pars tuberalis of adenohypophysis (Fig 5B). The spheric tumor shape of PCPs and upward compression of the optic chiasm were compatible with an origin in the pars tuberalis, which encircles the pituitary stalk (Fig 1B).

The posterior lobe develops from the downward evagination of neuroectodermal tissue originating from the diencephalon, called the "infundibulum." The neurohypophysis is embryologically and anatomically continuous with the hypothalamus. ${ }^{15} \mathrm{In}$ tracranial GCTs almost exclusively arise from the diencephalon, primarily the pineal region and hypothalamic-infundibular axis. These 2 regions are regulated by gonadotropins and connected via the accessory optic tract. ${ }^{16}$ It was proposed that GCTs originated 

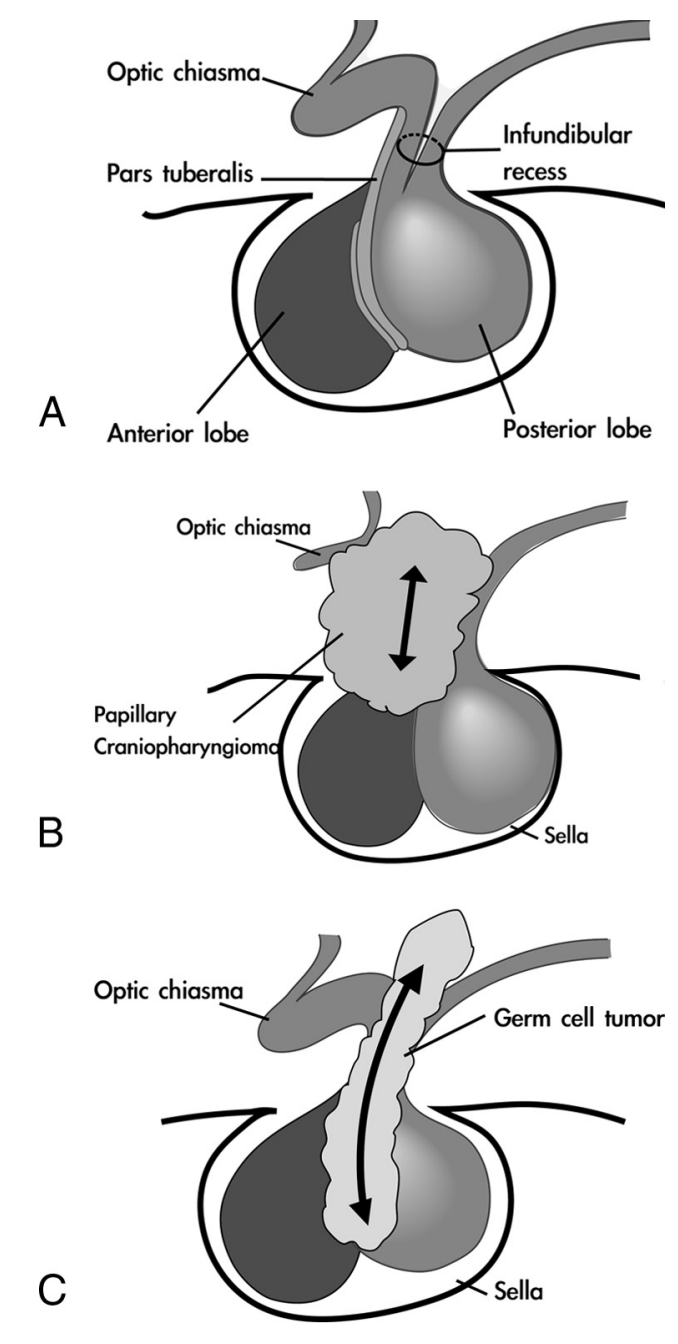

FIG 5. Hypothetic pathogenesis of suprasellar GCT and PCP. A, Normal anatomy of the sellar and suprasellar regions. The pars tuberalis, a small part of the pars anterior, extends ventrally and wraps around the pituitary stalk; the neurohypophysis is embryologically and anatomically continuous with the hypothalamus. $B$, The PCP originates from squamous epithelial cells in the pars tuberalis of the adenohypophysis and is located extraventricularly. C, The GCT originates from the hypothalamic-infundibular axis or pineal region. It is located intraventricularly and infiltrates along the infundibular recess down to the sellar region.

from the abnormal termination of primordial germ cell migration. Most hypophyseal GCTs present with infundibular thickening, which represents the only imaging findings in small tumors. ${ }^{5,17}$ This infundibular lesion can extend vertically into the sellar region along the pituitary stalk axis (Figs $3 A, 4 A,-B$, and $5 C$ ).

The pineal region is the most common location for GCTs, accounting for $40 \%-60 \% .{ }^{18}$ The suprasellar region is the second most common location of GCTs. Between $5 \%$ and $10 \%$ of intracranial germ cell tumors are found in both the pineal and suprasellar regions at diagnosis. This bifocal disease manifests mostly as germinoma, but its actual spreading or synchronous development remains unclear. ${ }^{7}$ We propose that suprasellar GCT presents more commonly as a spreading lesion from the primary pineal lesion than as a lesion with synchronous development or as a primary lesion. Some results of this study support our hypothesis: 1) In our 10 patients with suprasellar GCT who underwent surgi- cal management, the tumors were identified in the lower portion or floor of the third ventricle. This location implied that the suprasellar GCT had a close relationship with the intraventricular CSF flow dynamics and was influenced by gravity, particularly related to the pineal lesion with intraventricular involvement. 2) The transinfundibular tumor growth, diffuse thickening of the pituitary stalk, and downward infiltration of the pituitary gland on MR imaging suggested that the suprasellar GCT grew intraventricularly along the infundibular recess of third ventricle. This intraventricular location could also explain the common symptom of diabetes insipidus with suprasellar GCT because the hypothalamus has a close relationship with the third ventricle. By contrast, suprasellar PCPs originate outside the ventricle. Suprasellar PCPs presented with spheric tumor growth, displacement of the pituitary stalk, and compression of the optic chiasm. That PCPs had more upward growth toward the third ventricle (50\%) than GCTs $(24 \%)$ also suggests the extraventricular location and the influence of the hard structures of the tuberculum sellae and diaphragm sellae in their floor. 3) In half of the patients with GCT, the size of the pineal gland changed after low-dose radiation therapy. In the patients with recurrent GCT, tumor growth was revealed in the pineal gland and in the suprasellar region. The PCP group showed no significant interval change of the pineal gland, though the pineal gland was included in the field of radiation therapy. We suppose that the structure of the pineal gland in GCTs in the present study, even if the lesion is $<1 \mathrm{~cm}$, could be a part of the primary tumor. This radiosensitive tumor can shrink significantly from any low-dose irradiation, such as brain CT, and can regrow in some seeding areas. ${ }^{19,20}$ Due to gravity in upright position, the infundibular recess of the third ventricle is one of the most common sites for deposition of a seeding lesion. This seeding hypothesis of suprasellar GCT can corroborate the therapeutic results of pediatric GCT with low-dose irradiation including the ventricular system. ${ }^{20}$ Further study to compare the pathology of the pineal gland and suprasellar GCT will help confirm this hypothesis.

PCPs predominated in the older age groups, with bimodal distribution of craniopharyngiomas peaking in the fifth to sixth decades. ${ }^{6,21}$ Intracranial suprasellar GCTs were mostly diagnosed between 6 and 15 years of age. ${ }^{22}$ In the present study, the patients diagnosed with GCT were younger than those diagnosed with PCP; nevertheless, a 43-year-old man with GCT was identified. Regarding pediatric GCT, pineal tumors showed a male predominance, and suprasellar tumors were equally distributed between both sexes. ${ }^{7}$ We found male predominance in the adult patients of both groups in the present study. Both groups also presented with the common clinical symptoms of suprasellar tumors, including headache, visual field defects, decreased visual acuity, and hormone disturbance. ${ }^{23}$ However, GCTs commonly caused diabetes insipidus, which was consistent with hypothalamic-neurohypophyseal axis involvement. ${ }^{4}$

Several reports concerned optic pathway edematous changes associated with craniopharyngioma. ${ }^{24-26}$ Recently, this finding was also reported in other parasellar tumors, such as pituitary adenoma, GCTs, meningioma, and lymphoma. ${ }^{27}$ We also found optic pathway edema in both groups with no significant difference.

Only a few studies concerning MR imaging diffusion of PCPs have been published in the English literature. A case report of a 
huge craniopharyngioma showed high signal intensities on both DWI and ADC maps by its complex contents. ${ }^{28}$ In our study, the solid component of PCPs on DWI showed hypointense signals, presumably reflecting the character of this low-grade epithelial tumor. ${ }^{10,29}$ Intracranial GCTs were thought to demonstrate high nuclear-to-cytoplasmic ratios of tumor cells or high tumor cellularity, showing restricted diffusion on DWI. ${ }^{30}$ Routine axial whole-brain DWI may have problems in the evaluation of small suprasellar tumors because of scan gaps or artifacts of the skull base. We suggest sagittal DWI of the sellar region to improve the diagnosis of suprasellar tumors.

Although craniopharyngiomas are classified as grade I tumors by the World Health Organization, multiple recurrences and malignant transformations have been reported. Adhesion or encasement of adjacent cranial nerves or vessels also promotes the association of craniopharyngiomas with significant surgical morbidity. Aggressive surgical resection or conservative surgical treatment combined with adjuvant therapy remains a controversial therapeutic strategy. Regarding GCTs, germinomas respond well to radiation therapy, even without a pathologic diagnosis. Nongerminomatous GCTs require surgery or radiation therapy combined with chemotherapy. ${ }^{7}$ These diverse therapeutic strategies and outcomes highlight the importance of presurgical planning of these 2 unusual suprasellar tumors. According to our hypothesis of the extraventricular location of suprasellar PCPs, a transsphenoidal or subfrontal approach is a good option for tumor removal. An interhemispheric approach is appropriate for obtaining a pathologic diagnosis of the intraventricular location of suprasellar GCTs.

The major limitation of this study is the small number of cases of GCTs in the adult group. We included patients older than 16 years of age because they are beyond the common age distribution of pediatric GCT. ${ }^{22}$ The other limitation is that some cases of GCT did not have a pathologic diagnosis, including the interval change of pineal tissue on serial MR imaging. Further study comparing the pathologic and imaging changes of suprasellar and pineal structures will be helpful to clarify the origin and extension of these tumors and their best management.

\section{CONCLUSIONS}

Younger age, diabetes insipidus, MR imaging characteristics of restriction diffusion, and vertical infundibular extension favor the diagnosis of GCT. MR imaging findings of spheric shape without infundibular infiltration and without diffusion restriction provide clues to PCPs. We suggest that suprasellar GCT is possibly an intraventricular lesion originating from a pineal lesion or the hypothalamic-infundibular axis. Suprasellar PCPs originate from the pars tuberalis and are located outside the third ventricle. On the basis of clinical and MR imaging findings and these anatomic origins, appropriate pretreatment diagnosis and a surgical approach can be planned.

Disclosures: Feng-Chi Chang-RELATED: Grant: Taipei Veterans General Hospital (V103C-063, Cl-103-3) and the Ministry of Science and Technology (NSC 102-2314-B075-068), Comments: I am the Principal Investigator of these projects; Payment for Writing or Reviewing the Manuscript: Taipei Veterans General Hospital (V103C-063, Cl-103-3) and the Ministry of Science and Technology (NSC 102-2314-B-075-068), Comments: English editing of this submitted manuscript was supported by these projects. Wan-Yuo Guo-UNRELATED: Other: research collaboration with Siemens on topics other than the current submission under a contract between Taipei Veterans General Hospital and Siemens Healthcare.

\section{REFERENCES}

1. Dietemann JL, Cromero C, Tajahmady T, et al. CT and MRI of suprasellar lesions. J Neuroradiol 1992;19:1-22

2. Smith JK. Parasellar tumors: suprasellar and cavernous sinuses. Top Magn Reson Imaging 2005;16:307-15

3. Karnaze MG, Sartor K, Winthrop JD, et al. Suprasellar lesions: evaluation with MR imaging. Radiology 1986;161:77-82

4. Fujisawa I, Asato R, Okumura R, et al. Magnetic resonance imaging of neurohypophyseal germinomas. Cancer 1991;68:1009-14

5. Kanagaki M, Miki Y, Takahashi JA, et al. MRI and CT findings of neurohypophyseal germinoma. Eur J Radiol 2004;49:204-11

6. Zoicas F, Schofl C. Craniopharyngioma in adults. Front Endocrinol (Lausanne) 2012;3:46

7. Packer RJ, Cohen $\mathrm{BH}$, Cooney K. Intracranial germ cell tumors. Oncologist 2000;5:312-20

8. Sartoretti-Schefer S, Wichmann W, Aguzzi A, et al. MR differentiation of adamantinous and squamous-papillary craniopharyngiomas. AJNR Am J Neuroradiol 1997;18:77-87

9. Ogawa K, Shikama N, Toita T, et al. Long-term results of radiotherapy for intracranial germinoma: a multi-institutional retrospective review of 126 patients. Int J Radiat Oncol Biol Phys 2004;58:705-13

10. Fernandez-Miranda JC, Gardner PA, Snyderman CH, et al. Craniopharyngioma: a pathologic, clinical, and surgical review. Head Neck 2012;34:1036-44

11. Hamilton BE, Salzman KL, Osborn AG. Anatomic and pathologic spectrum of pituitary infundibulum lesions. AJR Am J Roentgenol 2007;188:W223-32

12. Van Effenterre R, Boch AL. Craniopharyngioma in adults and children: a study of 122 surgical cases. J Neurosurg 2002;97:3-11

13. Zada G, Lin N, Ojerholm E, et al. Craniopharyngioma and other cystic epithelial lesions of the sellar region: a review of clinical, imaging, and histopathological relationships. Neurosurg Focus 2010;28:E4

14. Sadler TW. Langman's Medical Embryology. Philadelphia: Lippincott Williams \& Wilkins; 2009:414

15. Nussey S, Whitehead S. Endocrinology: An Integrated Approach. Boca Raton: CRC Press; 2001:283-85

16. Glenn OA, Barkovich AJ. Intracranial germ cell tumors: a comprehensive review of proposed embryologic derivation. Pediatr Neurosurg 1996;24:242-51

17. Liang L, Korogi Y, Sugahara T, et al. MRI of intracranial germ-cell tumours. Neuroradiology 2002;44:382-88

18. McCarthy BJ, Shibui S, Kayama T, et al. Primary CNS germ cell tumors in Japan and the United States: an analysis of 4 tumor registries. Neuro Oncol 2012;14:1194-200

19. Yoneoka Y, Tsumanuma I, Jinguji S, et al. Synchronized multiple regression of diagnostic radiation-induced rather than spontaneous disseminated primary intracranial germinoma in a woman: a case report. J Med Case Rep 2011;5:39

20. Chen YW, Huang PI, Ho DM, et al. Change in treatment strategy for intracranial germinoma: long-term follow-up experience at a single institute. Cancer 2012;118:2752-62

21. Garnett MR, Puget S, Grill J, et al. Craniopharyngioma. Orphanet J Rare Dis 2007;2:18

22. Wong TT, Ho DM, Chang KP, et al. Primary pediatric brain tumors. Cancer 2005;104:2156-67

23. Huang BY, Castillo M. Nonadenomatous tumors of the pituitary and sella turcica. Top Magn Reson Imaging 2005;16:289-99

24. Higashi S, Yamashita J, Fujisawa H, et al. "Moustache” appearance in craniopharyngiomas: unique magnetic resonance imaging and computed tomographic findings of perifocal edema. Neurosurgery 1990;27:993-96 
25. Nagahata M, Hosoya T, Kayama T, et al. Edema along the optic tract: a useful MR finding for the diagnosis of craniopharyngiomas. AJNR Am J Neuroradiol 1998;19:1753-57

26. Hirunpat S, Tanomkiat W, Sriprung H, et al. Optic tract edema: a highly specific magnetic resonance imaging finding for the diagnosis of craniopharyngiomas. Acta Radiol 2005;46:419-23

27. Saeki N, Nagai Y, Matsuura I, et al. Histologic characteristics of normal perivascular spaces along the optic tract: new pathogenetic mechanism for edema in tumors in the pituitary region. AJNR Am J Neuroradiol 2004;25:1218-22
28. Sener RN, Dzelzite S, Migals A. Huge craniopharyngioma: diffusion MRI and contrast-enhanced FLAIR imaging. Comput Med Imaging Graph 2002;26:199-203

29. Kono K, Inoue $Y$, Nakayama K, et al. The role of diffusion-weighted imaging in patients with brain tumors. AJNR Am J Neuroradiol 2001;22:1081-88

30. Gavrilovic S, Lavrnic S, Thurnher M, et al. Proton MR spectroscopy and diffusion-weighted imaging of intracranial germ cell tumors: implications for differentiation from other lesions. European Journal of Radiology Extra 2011;79:e59-e64 